\title{
Improving Allocations through Revenue Redistribution in Auctions with Entry
}

\author{
Ruggiero Cavallo \\ Yahoo! Research, \\ 111 West 40th Street, \\ New York, NY 10018 \\ cavallo@yahoo-inc.com
}

\begin{abstract}
We consider a single-item private values auction setting with endogenous costly entry. It is known that for constant entry cost functions, the Vickrey auction has a unique symmetric Bayes-Nash "cutoff equilibrium" where only agents with value above some threshold enter. We extend this result to the case where cost is an arbitrary linear function of value. We then consider a class of strategyproof and no-deficit redistribution auctions that return Vickrey auction revenue back to participants (Bailey, 1997; Cavallo, 2006), demonstrating the existence of unique symmetric cutoff equilibria here also, with increased participation and expected allocation value (gross of costs) over the Vickrey auction. We show that when individuals' costs are purely a function of value, the equilibrium symmetric cutoff strategy achieved by the Vickrey auction is more efficient (net of costs) than any other cutoff level. But in the case where costs decrease as participation increases (e.g., when there is a search cost associated with obscure auctions), the Vickrey auction is inefficient and a redistribution auction dominates.
\end{abstract}

Keywords: auctions, redistribution mechanisms, entry costs, outside option, social welfare. 